International Journal of Modern Physics B

Vol. 33, No. 8 (2019) 1950053 (10 pages)

(C) The Author(s)

DOI: $10.1142 / \mathrm{S} 021797921950053 \mathrm{X}$

\title{
Delay-induced synchronization transition in a small-world neuronal network of FitzHugh-Nagumo neurons subjected to sine-Wiener bounded noise
}

\author{
Yuangen Yao, Ming Yi and Dejia Hou* \\ Department of Physics, College of Science, \\ Huazhong Agricultural University, \\ Wuhan, P. R. China \\ *hdj05@mail.hzau.edu.cn
}

Received 19 September 2018

Revised 9 December 2018

Accepted 27 December 2018

Published 16 January 2019

\begin{abstract}
Noise and delay are ubiquitous in brain and they have significant effects on neuronal network synchronization and even brain functions. Based on a small-world neuronal network of delayed FitzHugh-Nagumo (FHN) neurons subjected to sine-Wiener (SW) bounded noise, the effects of delay and SW noise on synchronization and synchronization transition are numerically investigated by calculating a synchronization measure $R$ and plotting spatiotemporal patterns. The phenomenon of delay-induced synchronization transition is observed as delay $\tau$ is increased. And large self-correlation time and strength of SW noise can increase the number of delay-induced synchronization transition. In addition, delay-induced synchronization transition is robust against the change of topology structure of neuronal network and this phenomenon becomes much easier to see for small nearest neighbors $k$ in the small-world network. Since synchronization transition may imply functional switch, our results may have important implications, and inspire future studies.
\end{abstract}

Keywords: Sine-Wiener bounded noise; synchronization transition; FitzHugh-Nagumo neuron.

PACS numbers: 05.45.-a, 05.40.-a

\section{Introduction}

Synchronization is a basis for a variety of brain functions, such as processing of sensory stimuli, $\stackrel{1,2}{r}$ formation of memory. $\cdot \frac{3,4}{r}$ The abnormal change of synchronization

This is an Open Access article published by World Scientific Publishing Company. It is distributed under the terms of the Creative Commons Attribution 4.0 (CC-BY) License. Further distribution of this work is permitted, provided the original work is properly cited.

* Corresponding author. 
may be related to the occurrence of disease, such as Parkinson's disease and epilepsy. $\frac{5}{5}$ Due to its importance, synchronization phenomena of coupled neurons in neuronal network have attracted extensive attention. There are many factors that affect synchronous behaviors of neuronal network, such as time delay, synapsis and autapse, topology structure of neuronal network, and omnipresent noise. Time delay is widespread due to finite propagation speed of information, $, 7,7$ and can induce synchronization transitions..$^{8-11}$ Neurons often connect to each other through synapsis and synaptic connections between different neurons are not constant but plastic through spike-timing-dependent plasticity. ${ }^{12,13}$ According to previous reports, the plasticity of synapsis can enhance synchronization and synchronization transitions. ${ }^{14}-16$ Especially, autapse as a special kind of synapsis generates a timedelayed self-feedback loop and can induce synchronization in a coupled neuronal network $\cdot \underline{17-19}$ And autaptic time delay can induce synchronization transition in single neuron and neuronal networks. ${ }^{19}-21$

Synchronized behaviors in neuronal networks are usually influenced by the topology structure of neuronal network. The topology structure of realistic neuronal networks in brain had been demonstrated to be small-world property. ${ }^{22,23}$ Realistic neuronal networks present highly clustered structure and sparsely long-range random connectivity between nonneighbor nodes, so that neuronal network with small-world property can support the required biological functions at the minimized costs. $\frac{23}{2}$ It has been reported that the increase of rewiring probability $p$ of small-world neuronal networks can improve synchronization ${ }^{24,25}$ and induce synchronization transition. ${ }^{8}$

Omnipresent noise is also a key factor for network synchronization. Counterintuitively, noise is not always disadvantageous to nervous system. ${ }^{26}$ The most famous examples are that noise can greatly amplify weak signal and thus improve signal detection, and that noise can enhance the reliability, regularity and synchrony of neuronal spiking. ${ }^{27}$ Many related studies have investigated the constructive roles of noise, ${ }^{28-30}$ but the concerned noise in most previous studies is regard as Gaussian noises. However, the misuse of Gaussian noise may lead to some unreasonable inferences in some real biological or physical system due to the unbounded nature of Gaussian noise. $\stackrel{31-34}{-3 n}$ One other hand, nonGaussian noise sources exist widely in many nonlinear systems, such as neurons of rat skin and crayfish. $\frac{35}{3}$ Based on these considerations, bounded noise attracts much attention and has been used widely in engineering, physical and biological systems. ${ }^{36}$ Remarkably, some interesting phenomena have been observed, such as bounded noise-facilitated spiral formation and breakup, $\frac{37,38}{3}$ and bounded noise-enhanced periodic signal detection. ${ }^{39}$ Sine-Wiener (SW) bounded noise is usually represented as a sinusoidal function with a constant amplitude and a random phase caused by a standard Wiener process. Reportedly, phase transitions in a time-delayed genotype selection model can be induced by SW bounded noise. ${ }^{40,41}$ In addition, SW bounded noise can also enhance weak periodic signal detection in the FitzHugh-Nagumo (FHN) neuron. $\underline{42}$ Cross-correlated sine-Wiener (CCSW) bounded noises can induce coherence resonance, $\stackrel{43}{ }$ and thus 
improve sub-threshold periodic detection in the FHN neuron. $\stackrel{44}{ }$ Moreover, CCSW bounded noises can also result in breakup of spiral wave and order-disorder spatial pattern transition. $\frac{45}{}$ In consideration of the importance of synchronization in brain functions, here we investigate the synchronization and synchronization transition in a small-world neuronal network of delayed FHN neurons subjected to SW bounded noise.

\section{Model and Simulation}

The dynamical properties of delayed FHN neurons subjected to SW noises in a small-world network are represented as ${ }^{10,46}$

$$
\begin{aligned}
\varepsilon \frac{d x_{i}(t)}{d t} & =x_{i}(t)-\frac{x_{i}^{3}(t)}{3}-y_{i}(t)+\sum_{j} g_{i j}\left[x_{j}(t-\tau)-x_{i}(t)\right] \\
\frac{d y_{i}(t)}{d t} & =x_{i}(t)+a+\eta_{i}(t) .
\end{aligned}
$$

Here, $x_{i}(t)$ denotes the membrane potential (fast variable) of the $i$ th neuron and $y_{i}(t)$ represents the corresponding recovery variable (slow variable). A small parameter $\varepsilon=0.01$ is used to carry out separation between fast and slow variables. And $g_{i j}=0.03$ stands for coupling strength between $i$ and $j$ neurons, while $g_{i j}=0$ indicates no connection between them. The value of $a=1.05$ ensures that only one stable attractor exists for the model, and that the model is excitable.

$\eta_{1}(t)$ is a SW bounded noise and its explicit definition is expressed as follows ${ }^{40,41}$ :

$$
\eta(t)=A \sin \left(\sqrt{\frac{2}{\lambda}} \omega(t)\right) .
$$

The strength of noise and self-correlation time of $\eta(t)$ are indicated as $A$ and $\lambda$, respectively. Unless otherwise indicated, $A$ is set to 0.1 throughout this paper. $\omega$ is a standard Wiener process generated in our numerical simulations according to following formula ${ }^{40,41}$ :

$$
\omega(t)=\omega(t-\Delta t)+\sqrt{-2 \Delta t \ln \chi_{1}} \cos \left(2 \pi \chi_{2}\right) .
$$

Here, $\chi_{1}$ and $\chi_{2}$ are two independent random numbers uniformly distributed on the unit interval, which are generated by the Box-Mueller algorithm. As previously reported, the average and auto-correlation function of SW bounded noise are given by $\underline{40,41}$

$$
\begin{aligned}
\langle\eta(t)\rangle & =0 \\
\left\langle\eta(t) \eta\left(t^{\prime}\right)\right\rangle & =\frac{A^{2}}{2} \exp \left(-\frac{t-t^{\prime}}{\lambda}\right)\left[1-\exp \left(-\frac{4 t^{\prime}}{\lambda}\right)\right], \quad t \geq t^{\prime}
\end{aligned}
$$


Equations (1) and (2) are discretized in our numerical simulations as follows:

$$
\begin{aligned}
x_{i}(t+\Delta t)= & x_{i}(t)+\frac{\Delta t}{\varepsilon}\left[x_{i}(t)-x_{i}^{3}(t) / 3-y_{i}(t)\right] \\
& +\frac{\Delta t}{\varepsilon} \sum_{j} g_{i j}\left[x_{j}(t-\tau)-x_{i}(t)\right], \\
y_{i}(t+\Delta t)= & y_{i}(t)+\left[x_{i}(t)+a\right] \Delta t+A \sin [\sqrt{2 / \lambda} \omega(t)] \Delta t .
\end{aligned}
$$

A synchronization measure $R$ is introduced to characterize the degree of network synchronization, and its distinct definition is given as follows ${ }^{10,47}$ :

$$
\bar{x}=\frac{1}{N} \sum_{i} x_{i}, \quad R=\frac{\left\langle\bar{x}^{2}\right\rangle-\langle\bar{x}\rangle^{2}}{\frac{1}{N} \sum_{i}\left(\left\langle x_{i}^{2}\right\rangle-\left\langle x_{i}\right\rangle^{2}\right)} .
$$

Here, $N=100$ is the number of neurons in the neuronal network. The symbol of $\langle\cdot\rangle$ denotes averaging over time of 1,000 time units (Note that there is no significant change in results if averaging over longer time). In addition, a sufficiently large time is used to discard the transient process before $R$ is calculated. It is evidently seen that $R$ is ranging between 0 (no synchronization) and 1 (perfect synchronization), and larger $R$ indicates better synchronization in neuronal network.

A small-world network is implemented below. Starting from a regular network, where each neuron connects to its $k=8$ nearest neighbor nodes, random longrange connections between nonnearest neighbor nodes are then introduced into the regular network by rewiring each link to another nonneighbor node randomly chosen with probability $p$. Therefore, the rewiring probability $p$ governs topology structure of network. Bigger $p$ denotes more long-range connections are added. Obviously, $p=0$ and $p=1$, correspond to the original regular network and a globally coupled network, respectively. Unless otherwise specified, $p=0.2$ is used throughout this study. The integral time step $\Delta t$ is fixed to 0.001 time units. The random initial conditions for $x, y$ and $\omega$ are adopted in our numerical simulations. Notably particularly, the use of different initial conditions and shorter time-step does not make significant changes in results.

\section{Results}

The dependence of synchronization measure $R$ with respect to time delay $\tau$ is presented in Fig. 1 for different self-correlation times $\log _{10}(\lambda)$ and strengths $A$ of SW noise. From this figure, it can be obviously observed that, with the increasing of time delay $\tau$, there exist obvious delay-induced synchronization transitions (Fig. 1). For small self-correlation time $\lambda$, such as $\log _{10}(\lambda)=-1, R$ keeps in a low value $(R \sim$ 0.02 ) when $\tau$ is small [Fig. 1(a)]; when $\tau$ is slightly greater than 1 , such as $\tau=1.25$ or 1.5 , moderate $R$ is obtained $(R \sim 0.45)$ [Fig. 1(a)]; finally, $R$ rapidly increases to a saturated value 0.998 when $\tau$ is larger than 1.75 [Fig. 1(a)]. For increasing self-correlation time $\lambda$, such as $\log _{10}(\lambda)=0,0.5$ and 1 , delay-induced multiple synchronization transitions can be observed [Fig. 1(a)]. The similar phenomena can 

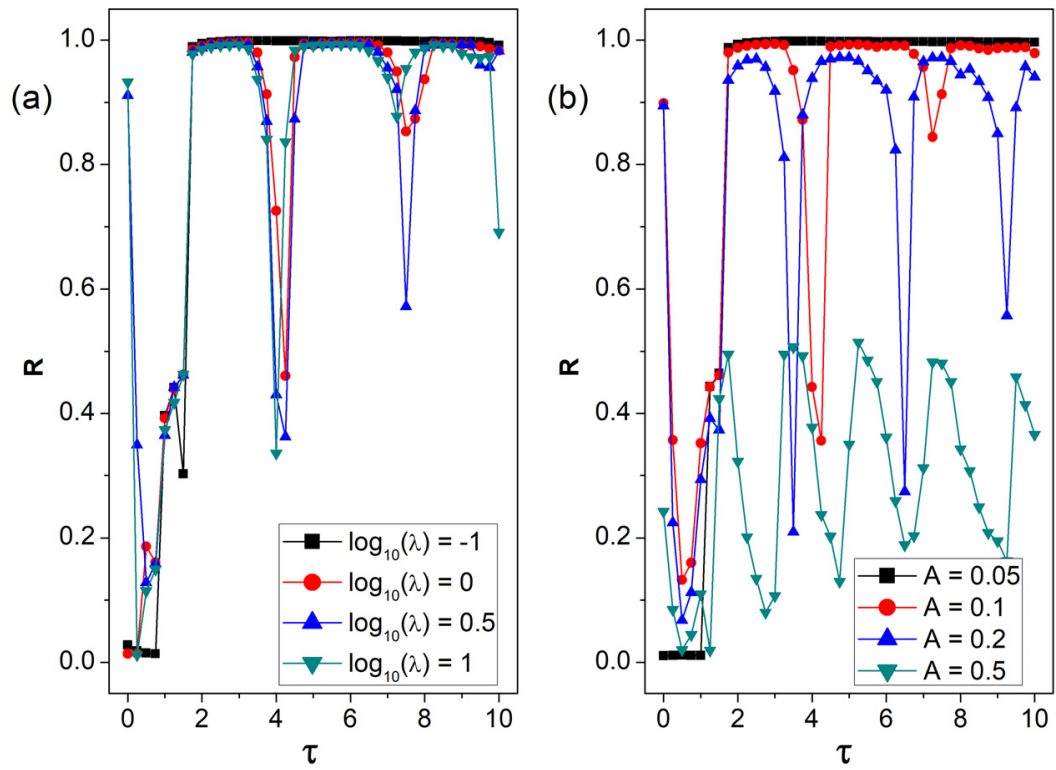

Fig. 1. (Color online) Dependence of the synchronization measure $R$ on time delay $\tau$ for different self-correlation times (a) and strengths (b) of SW bounded noise. (a) $A=0.1$ and (b) $\log _{10}(\lambda)=$ 0.5 .

also be obtained for increasing strength of SW noise. Therefore, compared with the case of small self-correlation time $\lambda$ ( $\operatorname{such}$ as $\log _{10}(\lambda)=-1$ ) or strength $A$ (such as $A=0.05)$ of SW noise, the increase of $\lambda$ or $A$ of SW bounded noise can result in more synchronization transition as time delay $\tau$ is increased.

In order to give a global view, we draw the contour plots of $R$ in $\log _{10}(\lambda)-\tau$ and $A-\tau$ planes. When $\log _{10}(\lambda)$ is fixed and is less than -0.3 , there is only an obvious synchronization transition as $\tau$ is increased [Fig. 2(a)]. Clearly, for large $\log _{10}(\lambda)$, the increase of $\tau$ may result in multiple synchronization transitions [Fig. 2(a)]. On the other hand, for small strength of noise, such as $A<0.05$, with the increase of time delay $\tau$, only one obvious synchronization transition can be observed [Fig. 2(b)]. For moderate strength of noise, delay-induced multiple synchronization transitions can be observed and the change of $R$ presents an oscillatory behavior as time delay $\tau$ is increased. Additionally, large strength of noise may destroy the network synchronization.

In order to comprehend delay-induced synchronization transition, the spatiotemporal patterns of membrane potential $x$ are drawn in Fig. 3. As shown in Fig. 3(a), without time delay $(\tau=0)$, firing activities of coupled neurons are regular. For $\tau=0.5$, the firing timings of coupled neurons are diverse and temporal regularity of coupled neurons is destroyed [Fig. 3(b)]. For $\tau=1.5$, the regularity of the temporal spiking behavior of coupled neurons is improved again [Fig. 3(c)], and the temporal regularity of coupled neurons can reach maximum at $\tau=2[\overline{\mathrm{Fig}}$. 3(d)]. For $\tau=4$, temporal regularity of coupled neurons is destroyed again [Fig. $3(\mathrm{e})]$. 

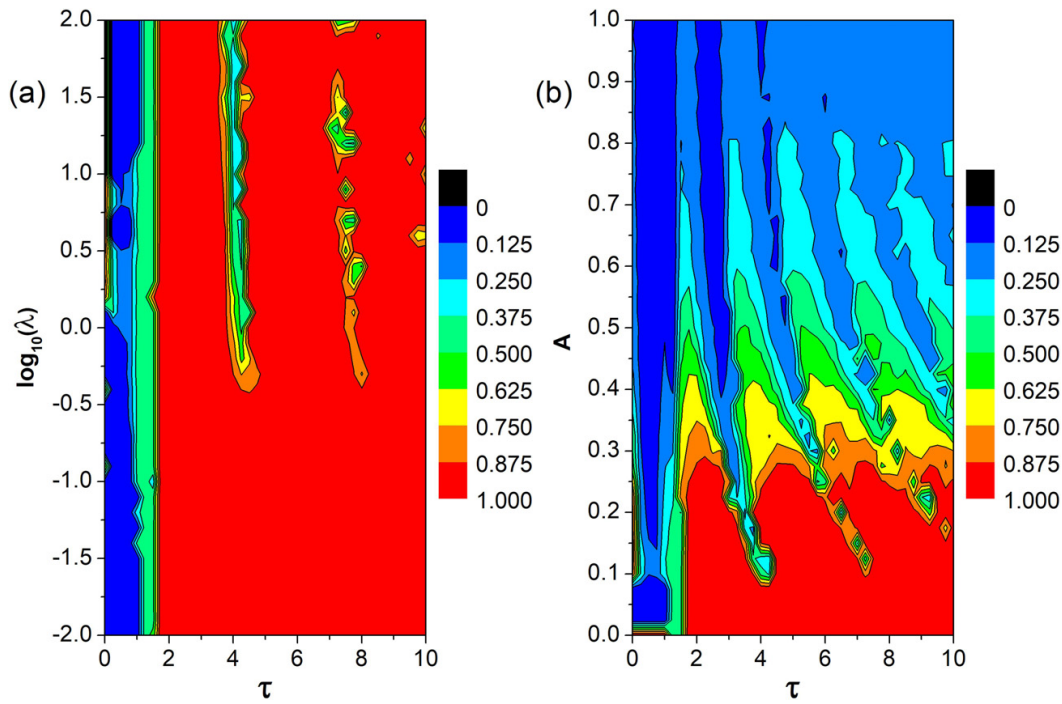

Fig. 2. (Color online) Contour plots of synchronization measure $R$ in the $\log _{10}(\lambda)-\tau$ and $A-\tau$ planes. (a) $A=0.1$ and (b) $\log _{10}(\lambda)=0.5$.

(a)

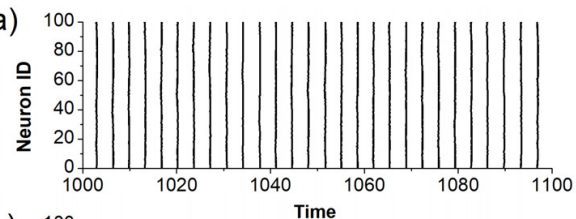

(c)

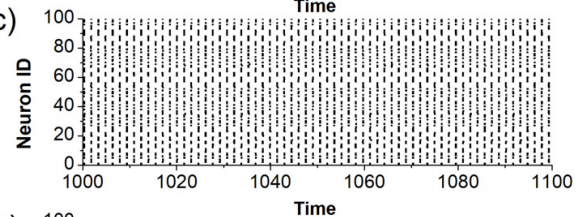

(e)

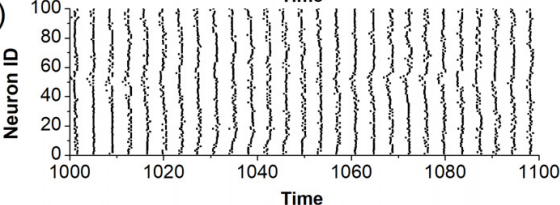

(b)

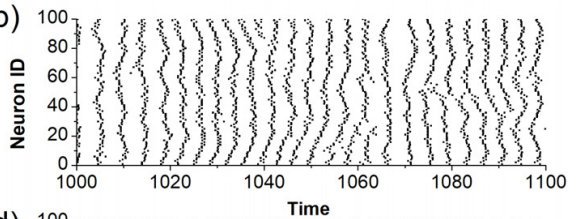

(d)

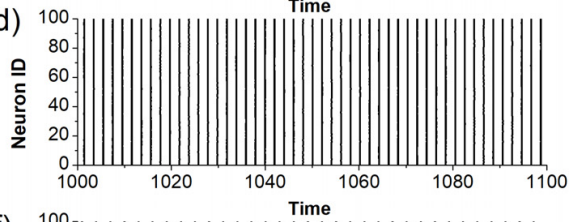

(f)

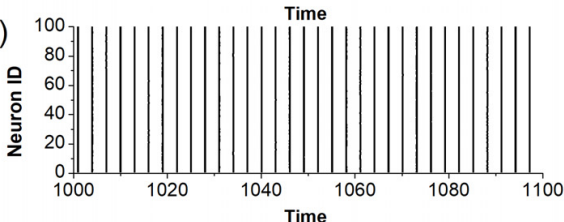

Fig. 3. Space-time plots of membrane potential $x$ for different time delay $\tau$. From (a) to (f), time delay $\tau$ is set sequentially to $0,0.5,1.5,2,4$, and $6 . A=0.1$ and $\log _{10}(\lambda)=0.5$.

However, for $\tau=6$, the regularity of the temporal spiking behavior of coupled neurons is regained [Fig. 3(f)]. Therefore, delay-induced synchronization transition is further confirmed through plotting spatiotemporal patterns. Moreover, we also draw the spatiotemporal patterns of membrane potential $x$ for different self-correlation times of SW noise when time delay $\tau$ is fixed to 4. As shown in Fig. 4, the regularity of the temporal spiking behavior of coupled neurons becomes increasingly 
(a)
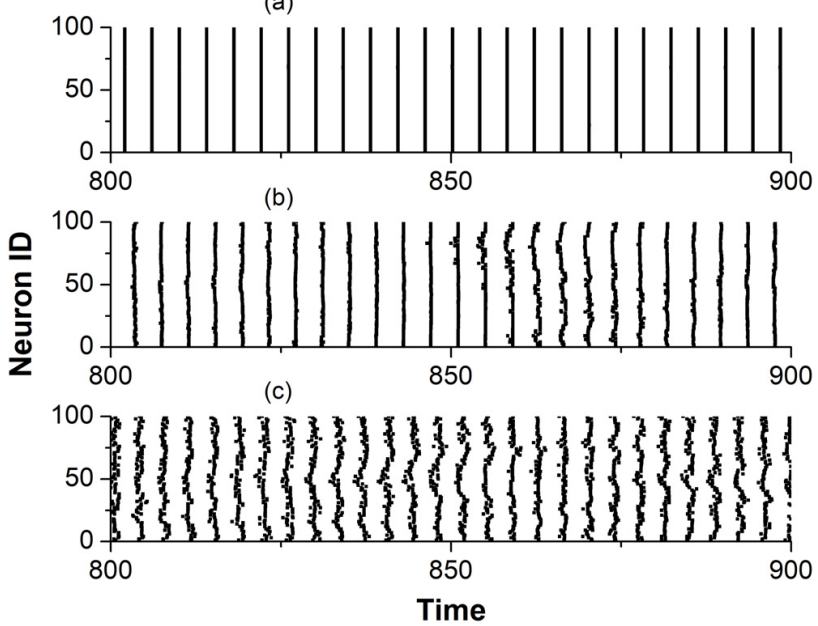

Fig. 4. Space-time plots of membrane potential $x$ for different self-correlation times of SW noise. From (a) to (c), $\log _{10}(\lambda)$ is set sequentially to $-2,0$, and $1 . A=0.1$ and $\tau=4$.

damaged as $\log _{10}(\lambda)$ is increased. This is also in accordance with the result obtained in Fig. 2(a). This result further illustrates that SW noise indeed has an important influence on synchronized behaviors of coupled neurons in small-world neuronal network.

We also investigate how the rewiring probability $p$ of small-world neuronal network influences the above-mentioned delay-induced synchronization transition. To
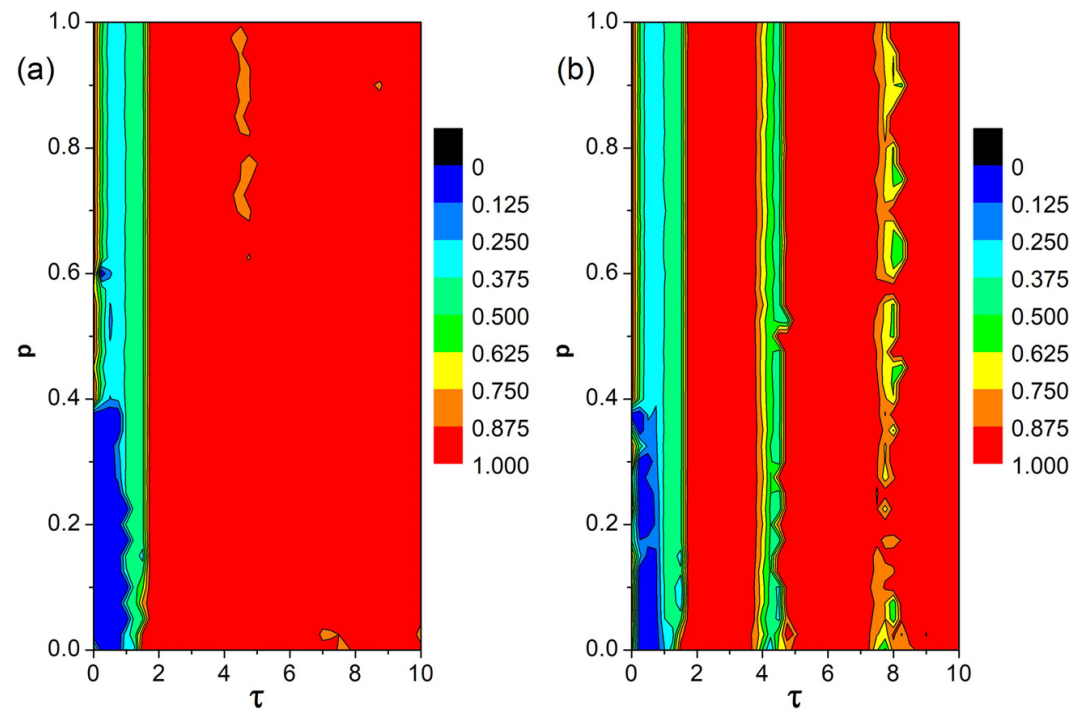

Fig. 5. (Color online) Contour plots of synchronization measure $R$ in the $\tau-p$ plane for two different self-correlation times of SW noise. $A=0.1$. (a) $\log _{10}(\lambda)=-1$ and (b) $\log _{10}(\lambda)=0$. 


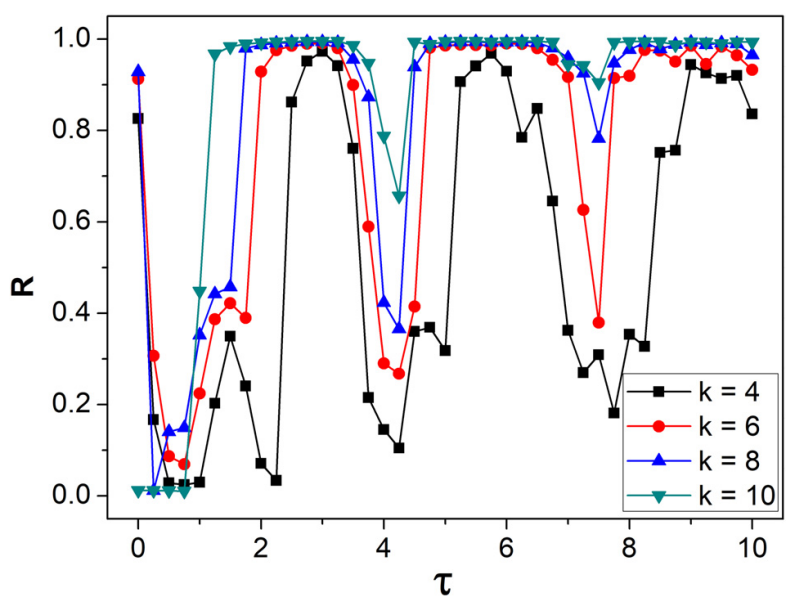

Fig. 6. (Color online) Dependence of the synchronization measure $R$ on time delay $\tau$ for different nearest neighbors $k$.

do this, we depict the dependence of $R$ on $\tau$ and $p$ for two different self-correlation times of SW noise in Fig. 5. Obviously, for each rewiring probability $p$, delay-induced synchronization transition can be observed (Fig. 5). Therefore, delay-induced synchronization transition is robust against the change of topology structure of neuronal network. In addition, compared with the cases of $\log _{10}(\lambda)=-1$ [Fig. 5(a)] and 0 [Fig. 5(b)], it is a robust conclusion in the small-world network that the increase of $\lambda$ can result in more delay-induced synchronization transitions. Finally, as illustrated in Fig. 6, the above-mentioned delay-induced multiple synchronization transition is more obvious for small nearest neighbors $k$ in small-world network.

\section{Conclusions}

In summary, the synchronization measure $R$ is calculated to quantitatively characterize the degree of the synchronization in a small-world network of delayed FHN neurons subjected to SW noise. The phenomenon of delay-induced synchronization transition is observed as time delay $\tau$ is increased. Large self-correlation time and strength of SW noise can increase the number of delay-induced synchronization transition. However, large strength of SW noise may reduce the degree of network synchronization. Through plotting the spatiotemporal patterns of firing activities of coupled neurons for several typical values of time delays $\tau$, the delay-induced synchronization transition is further confirmed. Furthermore, delay-induced synchronization transition is robust against the change of topology structure of neuronal network and this phenomenon of synchronization transition becomes much easier to see for small nearest neighbors $k$ in the small-world network.

It is widely accepted that synchronization is a reasonable mechanism for coding and transmitting information across different neurons, and synchronization transition may imply functional switch. Additionally, time delay and bounded noise 
indeed exist in the realistic neuronal networks. Consequently, our numerical study becomes significant, and we hope that the results reported here can inspire futurerelated studies. Besides noise and time delay, electromagnetic effect is also ubiquitous and it has been reported that electromagnetic effect has significant effects on electrical activities of neurons in some cases. $\stackrel{48-50}{-50}$ Therefore, the subjects of our future work is to investigate whether the results obtained in this paper are robust when neurons are subjected to combined SW noise and electromagnetic radiation.

\section{Acknowledgments}

This work was supported by the National Natural Science Foundation of China (Grant No. 31601071).

\section{References}

1. A. K. Kreiter and W. Singer, J. Neurosci: Official J. Society Neurosci. 16, 2381 (1996).

2. W. Singer, Annu. Rev. Physiol. 55, 349 (1993).

3. U. Rutishauser et al., Nature 464, 903 (2010).

4. N. Axmacher et al., Brain Res. Rev. 52, 170 (2006).

5. J. Premysl et al., J. Phys. 591, 787 (2013).

6. W. Dan, Z. Shiqun and L. Xiaoqin, EPL (Europhys. Lett.) 91, 40004 (2010).

7. D. Wu et al., Phys. Rev. E 84, 021102 (2011).

8. W. Qingyun et al., EPL (Europhys. Lett.) 83, 50008 (2008).

9. Q. Wang et al., Phys. Rev. E Stat. Nonlinear Soft Matter Phys. 80, 026206 (2009).

10. J. Tang et al., Phys. Rev. E Stat. Nonlinear Soft Matter Phys. 83, 046207 (2011).

11. D. Wu, S. Zhu and X. Luo, Physica A: Stat. Mech. Appl. 390, 1835 (2011).

12. L. Liu et al., Science 304, 1021 (2004).

13. N. Caporale and Y. Dan, Ann. Rev. Neurosci. 31, 25 (2008).

14. T. Nowotny et al., J. Neurosci. 23, 9776 (2003).

15. T. Pérez and A. Uchida, Phys. Rev. E Stat. Nonlinear Soft Matter Phys. 83, 061915 (2011).

16. Y. Gong, B. Wang and H. Xie, Bio Syst. 150, 132 (2016).

17. S. Guo et al., Complexity 2017, 13 (2017).

18. J. Ma et al., Neurocomputing 167, 378 (2015).

19. J. Ma et al., Chaos, Solitons Fractals 80, 31 (2015).

20. Q. Wang, Y. Gong and Y. Wu, Eur. Phys. J. B 88, 103 (2015).

21. C. Wang and J. Ma, Int. J. Mod. Phys. B 32, 1830003 (2018).

22. C. J. Honey et al., Proc. National Academy of Sciences 104, 10240 (2007).

23. D. S. Bassett and E. Bullmore, Neuroscientist 12, 512 (2006).

24. H. Hong, M. Y. Choi and B. J. Kim, Phys. Rev. E Stat. Nonlinear Soft Matter Phys. 65, 026139 (2002).

25. X. F. Wang and G. Chen, Int. J. Bifurcation Chaos 12, 187 (2002).

26. S. F. Traynelis and F. Jaramillo, Trends Neurosci. 21, 137 (1998).

27. G. B. Ermentrout, R. F. Galán and N. N. Urban, Trends Neurosci. 31, 428 (2008).

28. Q. Zhao, C. G. Yao and M. Yi, Eur. Phys. J. B 84, 299 (2011).

29. S. Liu et al., Physica A: Stat. Mech. Appl. 467, 184 (2017).

30. Q. Zhao et al., Front. Phys. 11, 110501 (2016).

31. A. D'Onofrio, Appl. Math. Lett. 21, 662 (2008). 
32. A. D'Onofrio and I. P. M. Tomlinson, J. Theor. Biol. 244, 367 (2007).

33. D. Li et al., Nonlinear Dyn. 70, 2237 (2012).

34. A. D'Onofrio and A. Gandolfi, Phys. Rev. E Stat. Nonlinear Soft Matter Phys. 82, 061901 (2010).

35. S. M. Bezrukov and I. Vodyanoy, Nature 378, 362 (1995).

36. A. D'Onofrio, Bounded Noises in Physics, Biology, and Engineering (Birkhäuser, Basel, 2013).

37. Y. Yao et al., Sci. Rep. 7, 43151 (2017).

38. Y. Yao et al., PLoS One 12, e0171273 (2017).

39. Y. Yao et al., Complexity 2018, 10 (2018).

40. L. J. Ning and P. Liu, Eur. Phys. J. B 89, 201 (2016).

41. H. Yang and L. J. Ning, Nonlinear Dyn. 88, 2427 (2017).

42. Y. Yao and J. Ma, Cogn. Neurodynamics 12, 343 (2018).

43. Y. Yao, M. Yi and D. Hou, Int. J. Mod. Phys. B 31, 1750204 (2017).

44. Y. Yao et al., Physica A: Stat. Mech. Appl. 492, 1247 (2018).

45. Y. Yao et al., Complexity 2018, 10 (2018).

46. R. FitzHugh, Biophys. J. 1, 445 (1961).

47. D. Gonze et al., Biophys. J. 89, 120 (2005).

48. M. Ge et al., Nonlinear Dyn. 91, 515 (2018).

49. Y. Xu et al., Neurocomputing 283, 196 (2018).

50. Y. Xu et al., Sci. Rep. 8, 1349 (2018). 